\title{
Therapeutic Effect of Intravenously Administered Autologous Adipose-Derived Stem Cells on Chronic Stage Stroke Patients
}

\author{
Masamitsu Ichihashi, MD, PhD ${ }^{1,2,3^{*}}$, Masaki Tanaka, MD, PhD ${ }^{1}$, Takashi lizuka ${ }^{1}$, Narumi \\ Nagoe $^{1}$, Youko Sato ${ }^{1}$, Hitomi Takahashi, Asami Ueda ${ }^{1}$, Hiroko Totsuka ${ }^{1}$, Narumi \\ Takanashi ${ }^{1}$, Takahito Nishikata, PhD ${ }^{4}$, Osamu Matsuo, MD, PhD ${ }^{5}$, Hideya Ando, PhD \\ and Ken-ichi Mizutani, PhD'
}

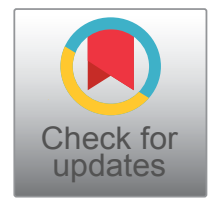

\author{
${ }^{1}$ Division of Stem Cell Therapy, BTR Arts Ginza Clinic, Japan \\ 2Laboratory of Stem Cell Biology, Graduate School of Pharmaceutical Sciences, Kobe Gakuin University, Japan \\ ${ }^{3}$ Anti-Aging Medical Research Center, Doshisha University, Japan \\ ${ }^{4}$ Frontier of Innovative Research in Science and Technology, Konan University, Japan \\ ${ }^{5}$ Faculty of Medicine, Kindai University, Japan \\ ${ }^{6}$ Department of Applied Chemistry and Biotechnology, Okayama University of Science, Japan
}

*Corresponding author: Masamitsu Ichihashi, MD, PhD, Division of Stem Cell Therapy, BTR Arts Ginza Clinic, 2-20-15-201 Shimbashi, Minato-ku, Tokyo 105-0004, Japan, Tel: 81-3-6228-5025, Fax: 81-3-6228-5026

\section{Abstract}

Background: Intravenous transplantation of mesenchymal stem cells derived from bone marrow and adipose tissue has been reported to be effective, safe and promising in the recovery of clinical outcomes of the stroke patients at acute and subacute stages, but clinical studies at chronic stages are still limited. It is reported that for better recovery of neurological dysfunction, cell therapy is recommended as early as possible after stroke onset. To our knowledge, clinical studies on stem cell therapy of stroke reported previously, did not describe the details of functional changes during and immediately after the end of intravenous stem cell transplantation.

Aim: In the present study, we aimed to clarify the effect of autologous adipose-derived stem cells (ADSC) on chronic stroke patients who were treated with ADSC intravenous drip-infusion later than 4 months after stroke onset. Further, we aimed to detect the time for functional recovery, and to find the clinical factors determining ADSC efficacy.

Patients and methods: We evaluated the effect of ADSC therapy on 21 stroke patients of 7 ischemic and 14 hemorrhagic damage, aged at 40-79 years at disease onset, focusing on 1) At the time lag between the day of stroke onset and ADSC therapy, 2) The age of stroke onset, 3) The number of transplanted cells, and 4) The time required to
\end{abstract}

confirm any first functional outcome of cell transplantation. ADSC were prepared from 2-3 small pieces of subcutaneous fat obtained from the abdominal skin. Materials cut into small pieces were placed on a scaffold and cultured with medium supplemented with $4 \%$ autologous serum for 11 to 13 days. After 21 day-culture, average number of $1.0 \times 10^{8}$ cells suspended in $200 \mathrm{ml}$ physiological saline were administered intravenously to the patients.

Results: Motor, sensory and cognitive functional recovery, and skin temperature increase were observed during and at quite early time after intravenous ADSC transplantation. Further, functional recovery evaluated by NIHSS was confirmed quite early time, around two-three hours after initiating ADSC treatment. Patients who treated with ADSC drip infusion within 6 months after stroke onset exhibited more apparent functional recovery compared with patients who were treated with ADSC later than 12 months after stroke onset. Age of patients, number of stem cell administered (from $0.6 \times 10^{8}$ to $1.4 \times 10^{8}$ ), and ischemic or hemorrhagic damage did not affect the efficacy of ADSC treatment.

Discussion and Conclusion: These results strongly indicate that functional recovery of stroke patients treated with ADSC may appear quite shortly after cell infusion, suggesting indirect interaction of ADSC and with intrinsic neuronal and vascular cells, and a pivotal role of extracellular vesicles

Citation: Ichihashi M, Tanaka M, lizuka T, Nagoe N, Sato Y, et al. (2020) Therapeutic Effect of Intravenously Administered Autologous Adipose-Derived Stem Cells on Chronic Stage Stroke Patients. Int J Stem Cell Res Ther 7:070. doi.org/10.23937/2469-570X/1410070

Accepted: December 08, 2020; Published: December 10, 2020

Copyright: (C) 2020 Ichihashi M, et al. This is an open-access article distributed under the terms of the Creative Commons Attribution License, which permits unrestricted use, distribution, and reproduction in any medium, provided the original author and source are credited. 
derived from ADSC during 90 minutes infusion. One of the most important factors determining the efficacy of ADSC infusion therapy of chronic stroke patients may be the time lag between the day of stroke onset and the day of ADSC therapy. Further studies remain to be conducted to recommend ADSC therapy as one of the most promising modalities for chronic stroke, especially to clarify the relation of brain damages site and efficacy of ADSC therapy.

\section{Introduction}

To overcome the limited application of thrombolytic or surgical endovascular therapies for patients with ischemic stroke, a number of preclinical studies of animal models and human clinical trials with intravenous transplantation of mesenchymal stem cells have been performed. Those results have been reported to be effective, safe and promising in the recovery of clinical outcomes of stroke patients at acute and subacute stages, but clinical studies at chronic stages are still limited [1-4]. Stem cells derived from autologous or allogenic bone marrow and/or subcutaneous adipocytes have been reported to be effective to recover sensory and motor functional outcomes mostly at subacute and rarely in chronic stages of stroke patients. Further, human adult stem cells have been reported to be highly resistant to spontaneous transformation, strongly indicating that mesenchymal stem cell transplantation may be a promising and safe therapeutic modality for strokes [5]. In 2011, Honmou, et al. [6] reported that a single intravenous transplantation of autologous human mesenchymal stem cells derived from bone marrow and expanded in human serum was effective and safe to reduce and improve the functional outcome in 12 stroke patients of ischemic grey matter, white matter and mixed lesions. They provided evidence supporting the feasibility and safety of autologous mesenchymal human stem cells for the treatment of stroke by demonstrating efficient outcomes by NIHSS (NIH stroke scale) and mRS (modified Rankin Scale). It has been reported that for better recovery of neurological dysfunction, cell therapy is recommended as early as possible after stroke onset [7].

To our knowledge, clinical studies on stem cell therapy of stroke reported to date have not described the details of functional changes of motor, sensory and cognitive functions during and immediately after the intravenous stem cell transplantation.

Stem cell therapy of strokes has been reported to promote the repair of the endogenous central nervous system and to stimulate neurorestorative mechanisms such as angiogenesis, neurogenesis, vascular remodeling, white matter remodeling and inflammatory and immune responses [8]. Recently, exosomes were suggested to mediate cell therapeutic effects on stroke by facilitating intercellular communications in a paracrine fashion, and to regulate intrinsic cell functions, so exosomes have been suggested to be a major mediator of stem cell therapy in stroke patients $[9,10]$.

In this clinical study, the efficacy of intravenous transplantation of autologous adipose-derived stem cells (ADSCs) for treating stroke patients at the chronic stage from 4 months to 8 years after the onset of stroke, on motor, sensory and cognitive functions during and immediately after cell infusion was evaluated by comparing NIHSS scores [11] just before the treatment, during 90 minutes treatment, and immediately after, one month, 6 month and 1 year after the treatment. The number of cells per treatment was approximately $1.0 \times$ $10^{8}$ cells. We evaluated the effects of ADSC therapy; 1 ) The time lag between the day of stroke onset and ADSC therapy, 2) The age of stroke onset, 3) The number of transplanted cells, and 4) The time required to confirm any first functional outcomes during and after the end of cell transplantation.

Informed consent was obtained from all stroke patients, and/or from next of kin in cases who were not able to write and/or understand the details of the stem cell treatment, after explaining the potential therapeutic effects including possible side effects. Our clinical study protocol was approved by our Clinic's Ethical Committee.

\section{Patients and Methods}

\section{Patients and evaluation of functional recovery}

Autologous adipocyte-derived mesenchymal stem cell (ADSC) therapy was performed to treat chronic ischemic and hemorrhagic patients who had a stroke onset more than 4 months and less than 6 years before the ADSC therapy. In the present clinical study, 21 cases of 7 ischemic and 14 hemorrhagic patients were included. The patient clinical characteristics are shown in Table 1. We analyzed the efficacy of ADSC therapy for 21 stroke patients who had severe to moderate neurological abnormalities of hemi-paralysis that remained after common therapy for the acute, sub-acute and chronic stages including rehabilitation. Most of the patients were treated with intravenous (IV) ADSCs after more than 6 months from the stroke onset, except four patients who were treated $43,52,82$ and 124 days after stroke onset (1 ischemic and 3 hemorrhagic stroke) (Figure 1). We report an un-blinded clinical study on 21 stroke patients who wanted to be treated with ADSCs after common therapy of stroke. The mean age of those subjects was 53.8 (range: 40 79 years) at stroke onset and 55.7 (40 80 years) at the day of treatment.

The severity of neuronal functions of the patients was evaluated by NIHSS, and the patients were categorized into two groups, one: $\geq 10$, and the other: $<10$. Further, to evaluate the efficacy of ADSC treatment in severity, NIHSS score changes after treatment were divided into three groups, $<1, \geq 1-<3$, and $\geq 3$. In addition, we observed the time needed for the first sign of improved motor neuronal and/or sensory function 


\begin{tabular}{|c|c|c|c|c|c|c|c|c|c|c|c|c|c|c|c|c|c|c|c|c|c|c|}
\hline \multirow{4}{*}{ 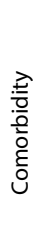 } & 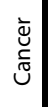 & I & 1 & 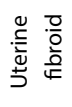 & 1 & I & 1 & 1 & I & 1 & 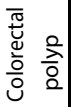 & I & 1 & 1 & I & 1 & I & 1 & 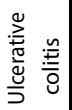 & 1 & I & I \\
\hline & $\vec{x}$ & + & 1 & 1 & 1 & 1 & \pm & 1 & 1 & 1 & 1 & 1 & 1 & 1 & 1 & 1 & 1 & + & 1 & 1 & 1 & + \\
\hline & 노 & 兵 & 1 & $\Phi$ & $\mp$ & + & + & $\mp$ & + & 王 & $\mp$ & $\Phi$ & $\mp$ & + & + & + & + & + & 1 & + & + & + \\
\hline & $\sum_{0}$ & 1 & 1 & 1 & 1 & 1 & 1 & 1 & I & I & 1 & I & 1 & 1 & 1 & 1 & 1 & 1 & + & 1 & + & + \\
\hline
\end{tabular}

\begin{tabular}{|c|c|c|c|c|c|c|c|c|c|c|c|c|c|c|c|c|c|c|c|}
\hline & 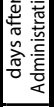 & $\stackrel{m}{m}$ & $\stackrel{n}{m}$ & $\bar{\sigma}$ & $\stackrel{\infty}{\sim}$ & $\stackrel{m}{m}$ & $\stackrel{m}{m}$ & in & 8 & 1 & б̄ & 1 & $\stackrel{n}{\wedge}$ & 1 & 1 & 1 & $\stackrel{m}{m}$ & స & \\
\hline & 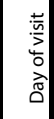 & 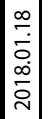 & $\begin{array}{l}\bar{i} \\
\stackrel{0}{0} \\
\infty \\
\grave{d}\end{array}$ & $\begin{array}{l}\stackrel{0}{ } \\
\stackrel{0}{ } \\
\infty \\
\stackrel{\infty}{\alpha}\end{array}$ & 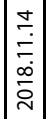 & 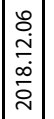 & $\left|\begin{array}{c}\infty \\
\stackrel{\infty}{\sim} \\
\infty \\
\infty \\
\tilde{N} \\
\end{array}\right|$ & 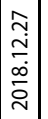 & 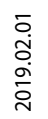 & 1 & 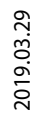 & 1 & 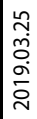 & 1 & 1 & । & $\begin{array}{l}\hat{N} \\
\tilde{o} \\
\stackrel{\dot{\alpha}}{0} \\
\dot{\alpha}\end{array}$ & 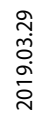 & 1 \\
\hline
\end{tabular}

\begin{tabular}{|c|c|c|c|c|c|c|c|c|c|c|c|c|c|c|c|c|c|c|c|c|}
\hline 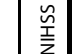 & $\infty$ & 1 & n & 1 & 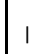 & 1 & 1 & 1 & 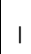 & 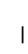 & 1 & 1 & 1 & 1 & 1 & 1 & 1 & 1 & 1 & 1 \\
\hline 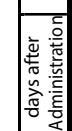 & 응 & 1 & $m$ & 1 & 1 & 1 & 1 & 1 & 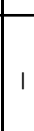 & 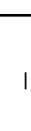 & 1 & 1 & 1 & 1 & 1 & 1 & 1 & 1 & 1 & 1 \\
\hline \begin{tabular}{|l|}
5 \\
\end{tabular} & $\infty$ & & $\widehat{\imath}$ & & & & & & & & & & & & & & & & & \\
\hline
\end{tabular}

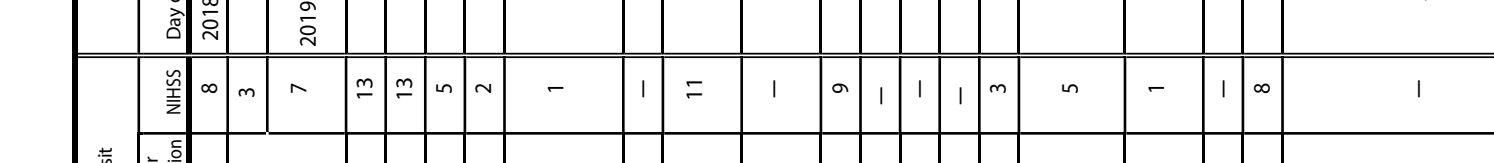

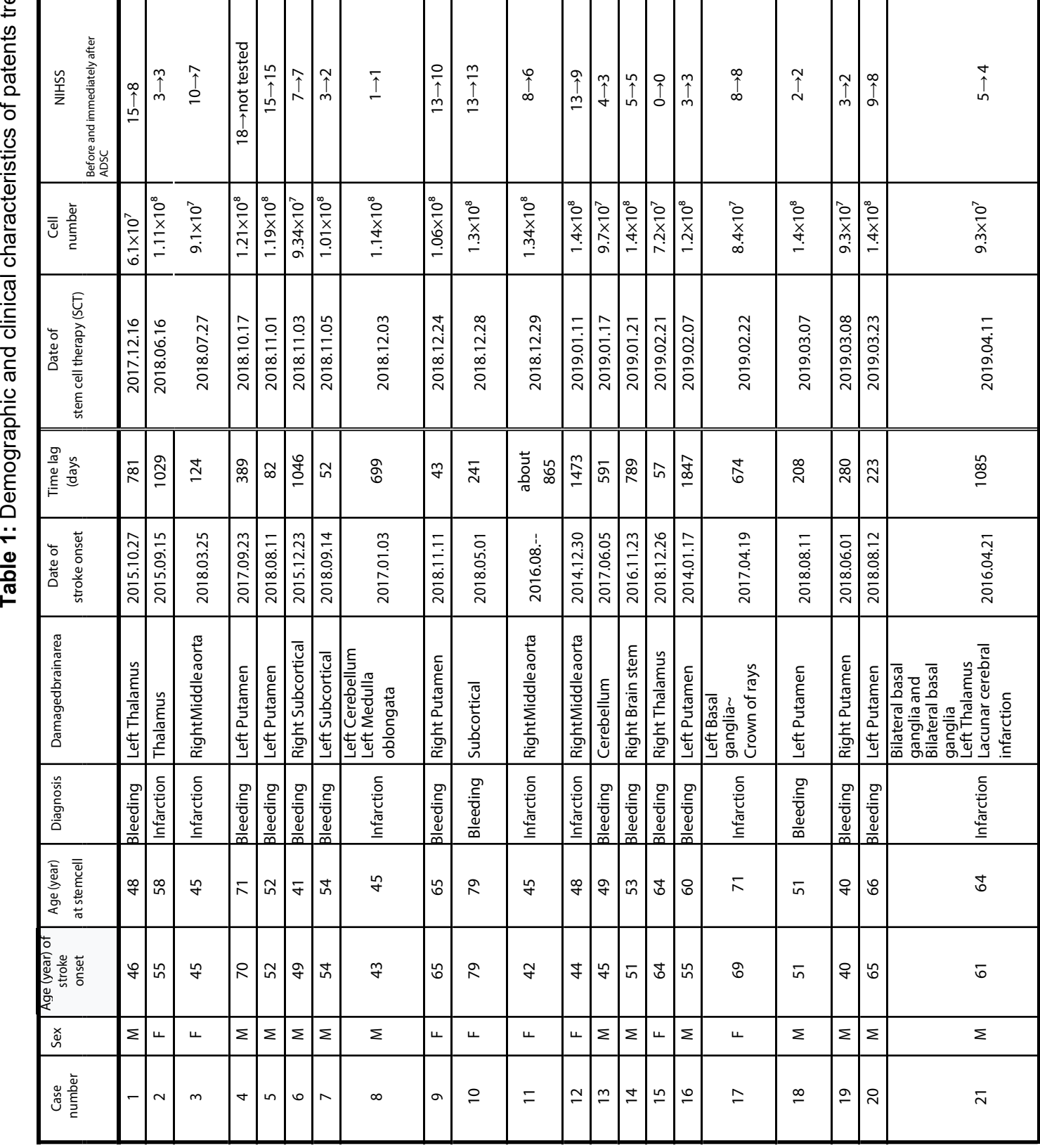




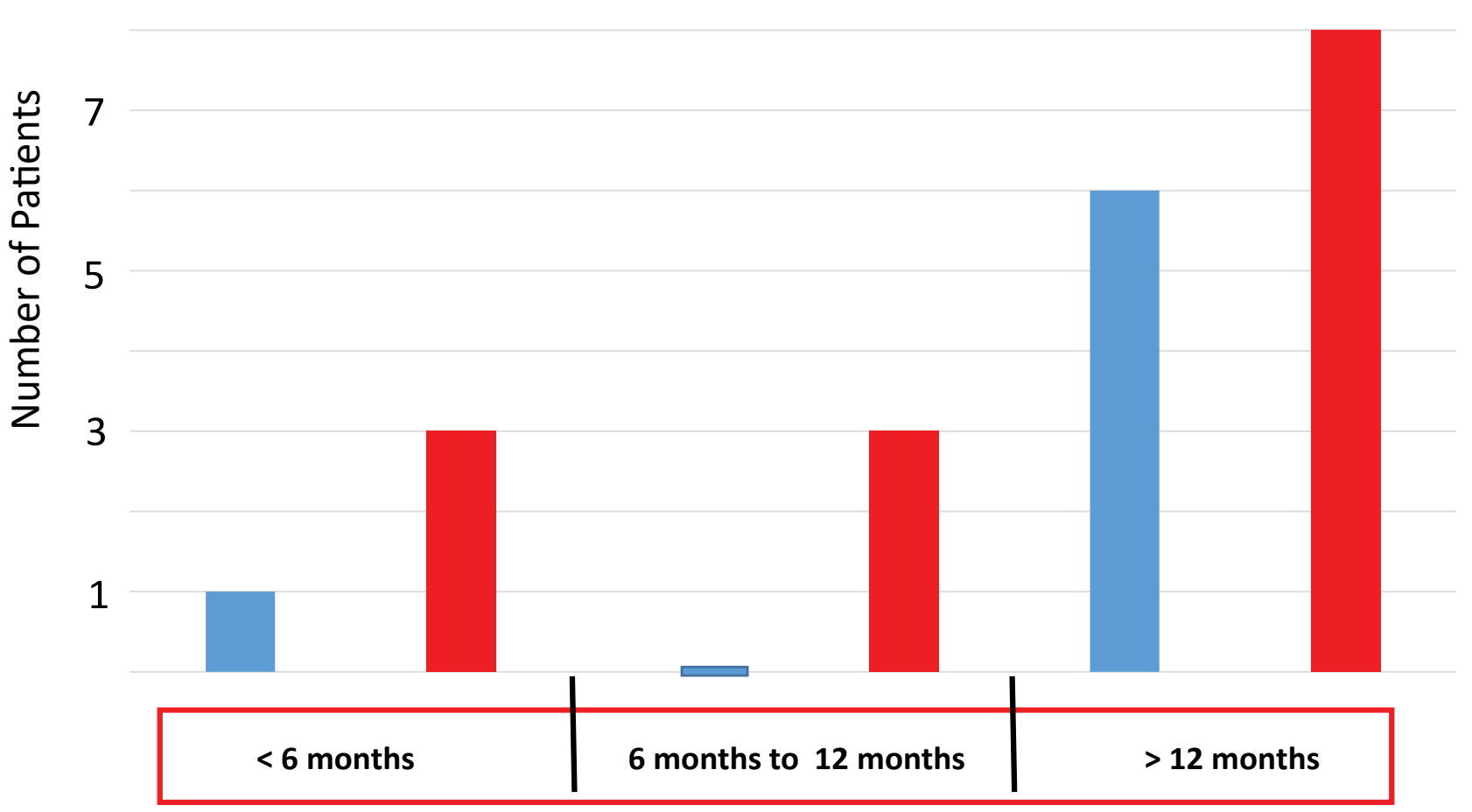

Figure 1: Number of patients with different time lags between the onset of stroke and ADSC therapy. The number of patients treated with autologous ADSCs before 6 months, between 6 and 12 months, and more than 12 months after the onset of stroke is shown. Blue and red bars indicate the number of patients suffering from infarction and hemorrhage, respectively.

in paralyzed extremities (erythema in numbed skin indicating a temperature increase), since we experienced a case who had a quite rapid recovery of more than 3 NIHSS score within two hours after the start of the drip infusion. After ADSC therapy, patients were advised to continue the rehabilitation, expecting further enhanced functional recovery. By examining patients the next day, one month, 6 months and one year after ADSC therapy, the functional recovery of each patient was evaluated, except some patients who could not visit our clinic and gave information of their functional recovery by telephone. A second course of ADSC therapy was performed for a few patients, at least more than 2 months after the first therapy. We excluded patients at the terminal stage of any kind of cancer.

\section{Preparation of autologous ADSC}

Patients were treated with a local anesthetic patch and injection on the skin approximately $10 \mathrm{~cm}$ to the side of the umbilicus, and 2 3 rice-sized pieces of sub-cutaneous fat tissues were surgically obtained from $0.7 \mathrm{~cm}$ incision. The fat tissues were cut into 15-20 small pieces and were placed on a scaffold, nonwoven fabric painted with hydroxy apatite (Bio Future Technologies, Tokyo) in culture dishes and were cultured at $5 \% \mathrm{CO}_{2}$, and $37^{\circ} \mathrm{C}$ in medium supplemented with $4 \%$ autologous serum for 11 to 13 days. Then, they were trypsinized (2.5/l-trypsin/1mmol/I-EDTA solution, Bio Future Technolgies, Tokyo) and reseeded in T75 flasks and further cultured for approximately 3 days in medium containing $2 \%$ serum, after which they were re-trypsinize and cultured in T300 flask (BM Equipment, Tokyo), and then trypsinized again and cultured for 3 days in Hyper Flasks (Corning Japan), before the final cell preparation for the treatment. At the day of transplantation, cells were trypsinized and washed 4 times with saline, then passed through two filters $(40 \mu \mathrm{m}$ and $100 \mu \mathrm{m})$ and an average number of $1.0 \times 10^{8}\left(0.6 \sim 1.4 \times 10^{8}\right)$ cells were prepared and suspended in $200 \mathrm{ml}$ saline and administered intravenously to the patients. The cells were further filtered with 180 $\mu \mathrm{m}$ pore size-mesh during drip infusion to remove clustered cells.

\section{Evaluation of skin temperature}

Change of the skin in patients who claimed coldness of their paralyzed extremities was evaluated by observing flushing and an improved warm feeling of the skin during and immediately after the ADSC therapy.

\section{Results}

\section{Effect of time lag between onset of the stroke and stem cell therapy on functional recovery}

Four patients who were treated with ADSC therapy within 6 months after the onset of stroke, and three patients who received ADSC therapy between 6 months and 12 months after the onset of stroke, showed apparent functional recoveries of greater than NIHSS 1, but among 14 patients who were treated with ADSCs more than 12 months after their stroke, 9 patients showed a recovery of greater than NIHSS 1 , and 5 patients showed 


\section{5}

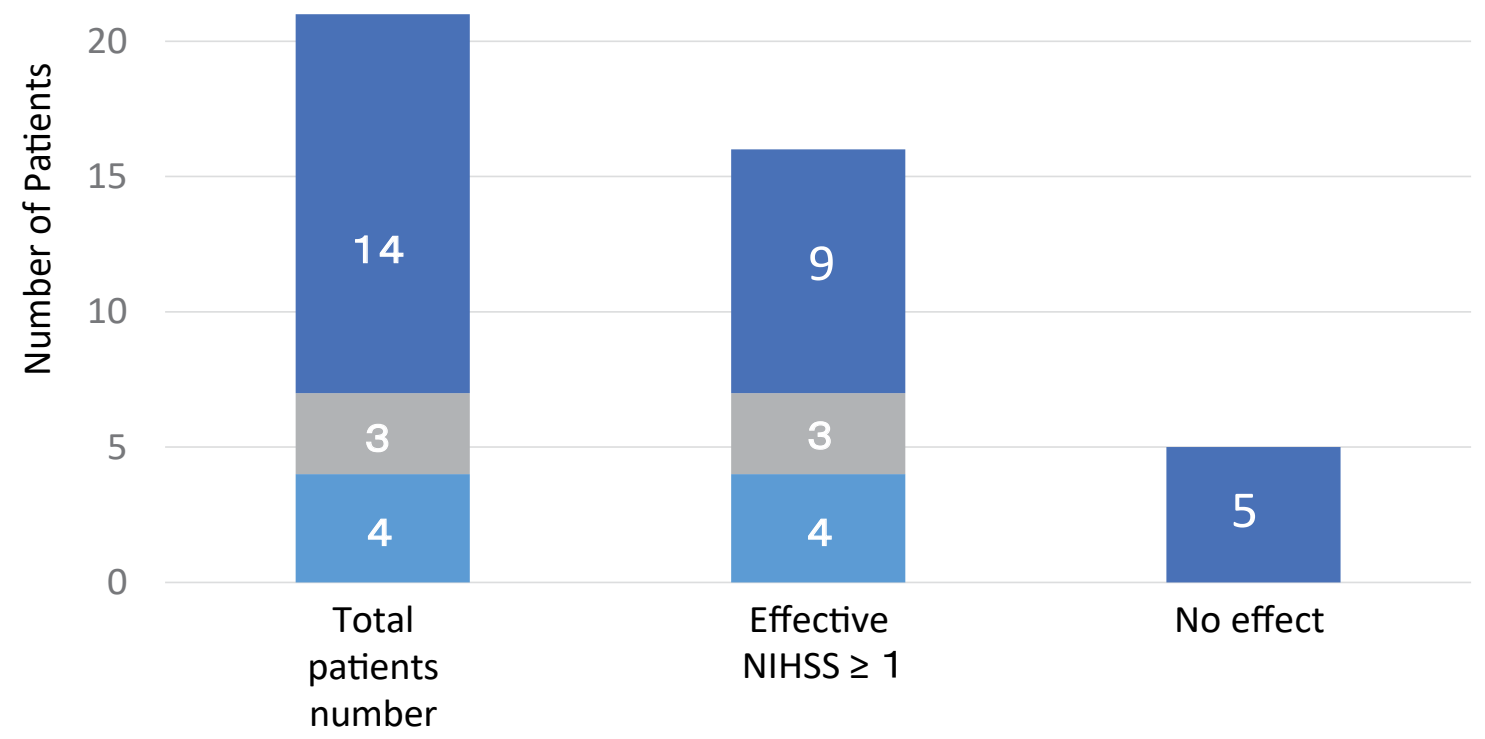

Figure 2: Effect of time lag between stroke onset and the time of ADSC therapy on functional recovery. The number of patients treated with ADSCs within 6 months ( $\square$ light blue), between 6 and 12 months ( $\square$ light gray) and after 12 months ( $\square$ dark blue) of stroke onset indicates that a shorter time lag after disease onset to ADSC treatment is more effective (NIHSS > 1 ), and one-third of patients treated with ADSCs later than 12 months showed little functional recovery.

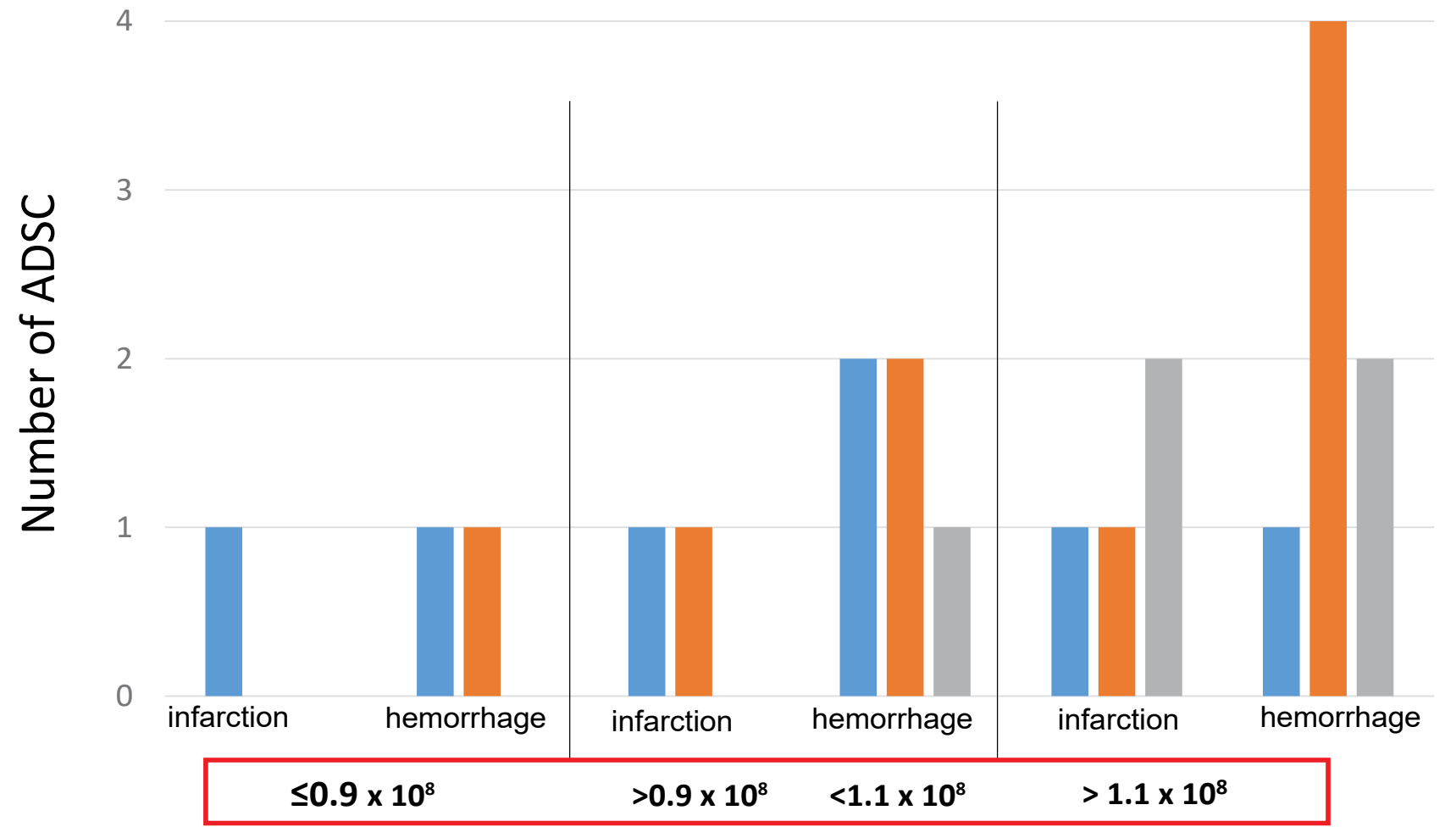

Figure 3: Effect of ADSC cell numbers of on the functional recovery of stroke patients. The effect of numbers of ADSCs was evaluated using NIHSS in 21 patients who suffered with infarction ( 7 cases) or hemorrhage (14 cases). Even patients receiving less than $0.9 \times 10^{8}$ ADSCs showed a similar efficacy compared to patients who were treated with more than $1.1 \times$ $10^{8}$ ADSCs. blue, orange and gray indicate numbers of patients who showed a functional recovery of HIHSS $>3,<$ 3 and no effect, respectively.

no recovery by NIHSS (Figure 2). These results suggest that a shorter time lag between stroke and ADSC therapy may be an important factor to gain functional recovery by the ADSC treatment.

\section{Effect of ADSC cell numbers administered on the functional recovery of stroke patients}

Among the three patients treated with less than 0.9 $\times 10^{8}$ cells $\left(0.6^{\sim} 0.8 \times 10^{8}\right)$, two showed a recovery great- 
er than or equal to 3 NIHSS, and one showed a recovery less than 3 NIHSS. Among the 7 patients treated with ADSC cell numbers between 0.9 and $1.1 \times 10^{8}, 3$ showed recoveries more than NIHSS 3 , while another 3 showed recoveries less than NIHSS 3 (Figure 3). Among the 11 patients who were infused with $1.4 \times 10^{8}$ ADSCs, two showed recoveries greater than NIHSS 3 , five showed recoveries less than NIHSS 3 , and four showed no functional recovery. These results indicate that ADSC cell numbers between $0.6 \times 10^{8}$ and $1.4 \times 10^{8}$ had similar recovery effects on patients with chronic stroke.

\section{Effect of age on cell therapy}

Among the 7 patients with greater than NIHSS 10, 3 patients younger than 50 , and one patient between 50 and 70 , and one patient older than 70 showed functional recovery of NISHH greater than 3 (Figure 4). One patient aged between 50 and 70 , and one patient older than 70 showed NIHSS less than 3 . Among the 14 patients with NIHS Slower than 10, one patient younger than 50 and one patient older than 70 showed NIHSS recovery greater than 3 . Three patients younger than 50 and 4 patients between 50 and 70 showed NIHSS recoveries less than 3 , while one patient younger than 50 and 4 patients at the age between 50 and 70 had no functional recovery. These results indicate that ADSC efficacy may not be affected by age at treatment.

\section{Time required for the first appearance of function- al recovery after intravenous ADSC}

Among the 21 patients, 9 showed functional recovery within 3 hours, and 5 patients had a functional recovery between 3 hours and 30 days (Figure 5). Two patients showed a functional recovery after more than one month. The remaining 5 cases did not show any recovery during the one year after ADSC therapy. These results strongly indicate that the functional recovery of stroke patients treated with intravenous infusion of ADSCs may appear quite shortly after cell infusion, suggesting indirect interactions of ADSCs with intrinsic neuronal and vascular cells.

\section{Effect of ADSC transplantation on skin tempera- ture of limbs with hemiplegia}

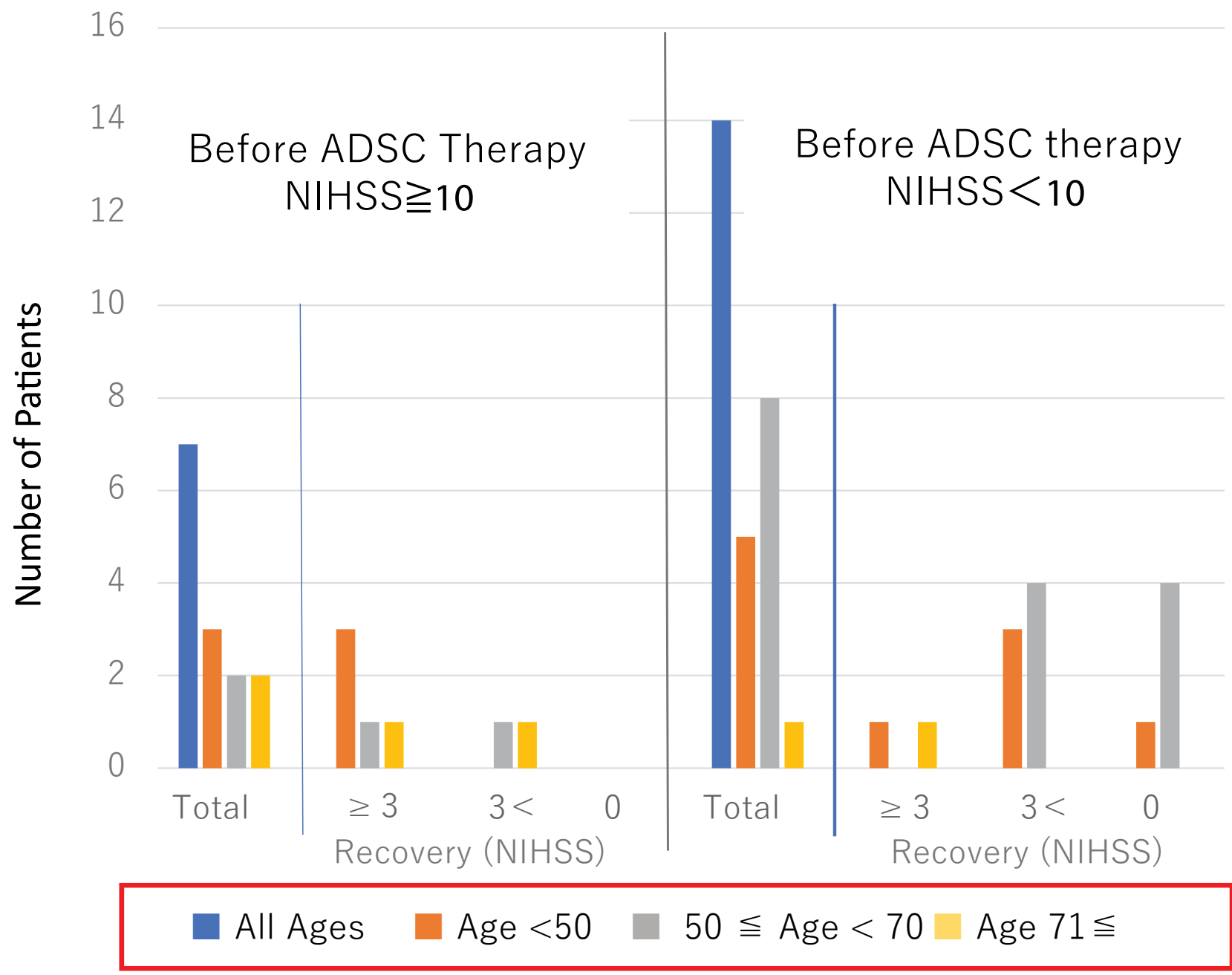

Figure 4: Effect of the age of patients on the functional recovery of ADSC therapy.

ADSC-treated patients were divided into two groups, one showing NIHSS recovery $>10$, and the other $<10$. indicate total number of patients, and —orange, gray and light orange bars indicate the ages younger than 50 , between 50 and 70 , and older than 70 years of age, respectively. 


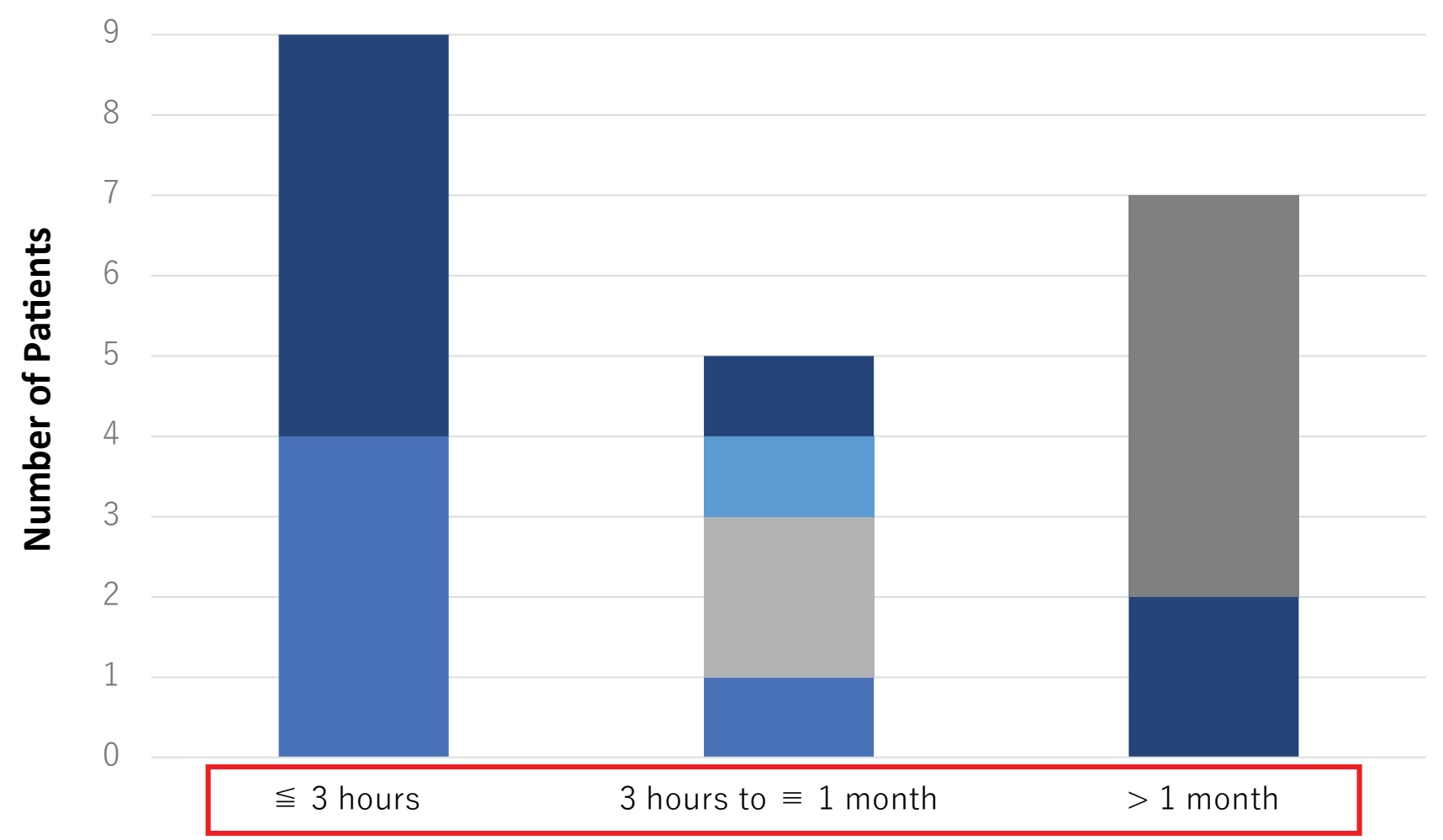

Figure 5: Time needed for the first appearance of functional recovery evaluated by NIHSS after ADSC therapy. Nine of the 21 cases showed a functional recovery before 3 hours after the ADSC therapy, 5 cases showed a functional recovery after 3 hours but earlier than one month, and 2 cases showed a functional recovery later than one month. Five cases had no recovery at one year after ADSC therapy.

NIHSS before ADSC $\geqq 10$, recovery of NIHSS after ADSC $\geqq 3$, NIHSS $\geqq 10$ recovery of NIHSS after ADSC $<3$

NIHSS before ADSC $<10$, recovery of NIHSS after ADSC $\geqq 3$,

NIHSS before ADSC $<10$, recovery of NIHSS after ADSC 0

Case 3, who had cold skin of limbs with hemiplegia recovered the skin temperature to a level similar to healthy limb's skin, during the ADSC transplantation. We experienced a few cases who exhibited reddish skin during or at the end of the ADSC infusion. That result suggests that ADSC therapy may exert an effect on blood circulation recovery via small molecules excreted as extracellular vesicles from cultured cells, since recovery within a few hours after the infusion is too soon for the infused cells to have reached to the damaged brain area. To confirm and elucidate the effects of intravenous ADSC therapy on the skin temperature of stroke patients, we need to measure the skin temperature of many more stroke patients in the future.

\section{Discussion}

During the last two decades, one effective ischemic stroke therapy has been shown to be the intravenous administration of tissue plasminogen activator which has been approved by the FDA in the USA and in other developed countries, although its efficacy has been limited due to its narrow time window. Endovascular therapies utilizing intra-arterial mechanical or chemical thrombolysis also improve outcomes, but therapeutic applications are again limited due to a narrow time window. After the acute time period, focused physical rehabilitation of the injured area is the primary current therapy that is proven to be effective for strokes $[12,13]$.

Re-organization of the cortex has been observed with rehabilitation in pre-clinical models as well as in humans [14]. While rehabilitation can be effective and encouraging results have been reported [15], the extent of neurologic recovery is still limited and novel approaches to enhance the endogenous regenerative abilities are required. In laboratory studies, stem cell therapy has proven to be a safe and potential method to regenerate an injured brain beyond the acute phase of stroke, even at the chronic stage, although limited clinical trials have shown that stem cell transplantation is effective for treating stroke patients at the chronic stage. Bhasin, et al. reported that autologous mononuclear stem cell transplantation had safe and slightly therapeutic effects for chronic stroke patients [16]. However, that study did not mention any functional recovery at an earlier time, around one or two hours after the treatment.

Surprisingly, we observed a significant motor function improvement in paralyzed upper and lower limbs in three patients at the chronic stage immediately after the 90 minute-drip-infusion of autologous ADSCs. In Case 1 , NIHSS decreased dramatically after the treatment from score 15 before treatment, to score 12 immediately af- 
ter, and score 8 one day after the ADSC transplantation, indicating that intravenous ADSC therapy of stroke patients at the chronic stage may recover functional outcomes during and shortly after the therapy. Further, in this case, the speech defect attributable to the disorder of phonation was improved immediately after cell infusion. In Case 3, in addition to an immediate significant recovery of the movement of paralyzed limbs, the paralyzed tactile sensation in both the upper and lower extremities was significantly recovered to almost a normal level nearly one hour after starting of the stem cell infusion and the effect continued until one month after the stem cell therapy when the patient visited our clinic for confirmation of the therapeutic effect. Further, the skin of paralyzed limbs became reddish at elbow, knee and foot joints, in accordance with the patient's claim of a hot feeling of the limbs. In Case 9, a 65-year-old female who took ADSC therapy 43 days after the onset of stroke, exhibited an apparent movement recovery of her lower extremity and a recovery of her skin touch sensation immediately after ADSC infusion, suggesting the importance of cell therapy timing after the onset of stroke, but not of the onset age of the disease. Further, 11 cases, including these three cases, exhibited a functional recovery by NIHSS within three hours after initiating cell therapy, indicating that ADSC therapy may efficiently recover neurological functions during the first 3 hours, possibly through the functional stimulation of endogenous neuronal cells via the extracellular vesicles excreted during infusion. This suggests a low possibility of the replacing damaged neuronal cells with the intravenously transplanted stem cells, at least during the first three hours after the end of cell therapy. Four cases in whom functional recovery was not observed during the 3 hours after cell infusion showed a functional recovery of NIHSS, 2, 2, 3 and 1 respectively, at their visit to the Clinic one month after cell therapy. That suggests possible neuronal function recovery due to continued rehabilitation after a single stem cell therapy.

One among the 6 cases who had no early recovery exhibited a functional recovery of NIHSS but the remaining 5 cases did not show any functional recovery at one year after cell therapy.

The dramatic early recovery of motor and/or sensory functions observed within two to three hours after ADSC treatment in 11 patients suggests that extracellular factors secreted from stem cells may play a pivotal role in the activation of motor and sensory functions of the extrapyramidal system.

To clarify which factors affect the therapeutic efficacy of ADSCs, such as the stem cell characteristics in each patient, the volume and/or location of the damaged brain of the stroke patient, and general health conditions including co-morbidities, diabetes and hypertension and medication, further clinical studies based on detailed information of each patent are required.
In order to perform successful clinical trials with optimal efficacy for stem cell therapy of chronic stroke patients, it is important to report the efficacy and safety of clinical stroke cases treated with stem cells at a later chronic stage whose functional recovery is considered to have reached almost a plateau after conventional treatments including rehabilitation. Even if a study is not conducted using a placebo control, the results are expected to contribute to better establish stem cell therapy of stroke patients through detailed therapeutic information based on numerous cases with diverse clinical characteristics.

Finally, our study strongly suggests that the early effect of intravenous transplantation of ADSCs in chronic stroke patients may be caused by extracellular vesicles released from cultured ADSC suspended in $200 \mathrm{ml}$ saline during 60 minute-cell preparation and the 90 minute-intravenous infusion times, which may contribute to recovering neuronal functions, and other effect, such as skin temperature increase. The functional recovery observed more than one month after cell therapy may be induced by interactions of recruited infused stem cells with intrinsic regenerative cells located in the injured brain tissues, and the rehabilitation performed after the cell therapy. Since we have experienced ADSC therapy of only a small number and a short follow-up term of chronic stroke patients, and we have not yet studied the fate of infused ADSCs, it is not clear whether the infused ADSCs directly communicate with neuronal cells in and around damaged brain tissues and/or have differentiated into neuronal and vascular cells at functional recovery observed more than one month after treatment. Further pre-clinical and careful clinical studies are required to clarify the efficacy of extracellular vesicles without stem cells, since exosomes and other small biological molecules that are free from pulmonary occlusions commonly induced by cell therapy are expected to be safer therapy for stroke and other diseases with vascular and neuronal disturbances in brain [17-21].

The number of stroke patients in our study is too small to conclude that intravenous ADSC therapy induces a functional recovery shortly after the treatment in most patients and is influenced by the time lag between the stroke onset and ADSC therapy, but not by the age of patients and the number of ADSCs. Further studies remain to be conducted to recommend ADSC therapy as one of the most promising modalities for treating chronic stroke patients.

\section{References}

1. Oh Young Bang, Jin Soo Lee, Phil Hyu Lee, Gwang Lee (2005) Autologous mesenchymal stem cell transplantation in stroke patients. Ann Neurol 57: 874-882.

2. Jeong Chan Ra, II Seob Shin, Sang Han Kim, Sung Keun Kang, Byeong Cheol Kang, et al. (2011) Safety of intravenous infusion of human adipose tissue-derived mesenchymal stem cells in animals and humans. Stem Cells Dev 20: 1297-1308. 
3. Ashu Bhasin, MV Padma Srivastava, S Senthil Kumaran, Sujata Mohanty, Rohit Bhatia, et al. (2011) Autologous mesenchymal stem cells in chronic stroke. Cerebrovasc Dis Extra 1: 93-104.

4. Diamandis T, Borlongan CV (2015) One, two, three steps towards cell therapy for stroke. Stroke 46: 588-591.

5. Jin Soo Lee, Ji Man Hong, Gyeong Joon Moon, Phil Hyu Lee, Young Hwan Ahn, et al. (2010) A long-term follow-up study of intravenous autologous mesenchymal stem cell transplantation in patients with ischemic stroke. Stem Cells 28: 1099-1106.

6. Osamu Honmou, Kiyohiro Houkin, Takuya Matsunaga, Yoshiro Niitsu, Sumio Ishiai, et al. (2011) Intravenous administration of auto serum-expanded autologous mesenchymal stem cells in stroke. Brain 134: 1790-1807.

7. Mark A Eckert, Quynh Vu, Kate Xie, Jingxia Yu, Wenbin Liao, et al. (2013) Evidence for high translational potential of mesenchymal stromal cell therapy to improve recovery from ischemic stroke. J Cere Blood Flow Metab 33: 13221334.

8. Adriana Carol Eleonora Graziano, Rosanna Avola, Vincenzo Perciavalle, Ferdinando Nicoletti, Gianluca Cicala, et al. (2018) Physiologically based microenvironment for in vitro neural differentiation of adipose-derived stem cells. $\mathrm{W} \mathrm{J}$ Stem Cells 26: 23-33.

9. Chen JC, Chopp M (2019) Exosome therapy for stroke. Stroke 49: 1083-1090.

10. Thorsten R Doeppner, Josephine Herz, André Görgens, Jana Schlechter, Anna-Kristin Ludwig, et al. (2015) Extracellular vesicles improve post-stroke neuroregeneration and prevent postischemic immunosuppression. Stem Cells Transl Med 4: 1131-1143.

11. Laura Otero Ortega, Fernando Laso García, Mari Carmen Gómez-de Frutos, Blanca Fuentes, Luke Diekhorst, et al. (2019) Role of exosomes as a treatment and potential biomarker for stroke. Transl Stroke Res 10: 241-249.

12. Janne Marieke Veerbeek, Erwin van Wegen, Roland van Peppen, Philip Jan van der Wees, Erik Hendriks, et al. (2014) What is the evidence for physical therapy poststroke? A systematic review and meta-analysis. PLoS One 9: e87987.
13. Joachim Liepert, Ina Unde, Sylvia Gräf, Ottmar Leidner, Cornelius Weiller (2001) Motor contex plasticity during forced-use therapy in stroke patients: A preliminary study. $\mathrm{J}$ Neurol 248: 315-321.

14. Brian J Hoare, Jason Wasiak, Christine Imms, Leeanne Carey (2007) Constraint-induced movement therapy in the treatment of the upper limb in children with hemiplegic cerebral palsy. Cochrane Detabase Syst Rev 2: CD004149.

15. A Bhasin, MV Srivastava, R Bhatia, S Mohanty, SS Kumaran, et al. (2012) Autologous intravenous mononuclear stem cell therapy in chronic ischemic stroke. J Stem Cells Regener Med 3: 181-189.

16. Janne M. Veerbeek, Muriel Koolstra, Johannes C.F. Ket, Erwin E.H. van Wegen, Gert Kwakkel (2011) Effects of augmented exercise therapy on outcome of gait and gait-related activities in the first 6 months after stroke: A meta-analysis. Stroke 42: 3311-3315.

17. Rebekah M Samsonraj, Michael Raghunath, Victor Nurcombe, James H Hui, Andre J van Wijnen, et al. (2017) Concise review: Multifaceted characterization of human mesenchymal stem cells for use in regenerative medicine. Stem Cells Transl Med 6: 2173-2185.

18. Rita Catarina Assunção-Silva, Bárbara Mendes-Pinheiro, Patrícia Patrício, Leo A Behie, Fábio Gabriel Teixeira, et al. (2018) Exploiting the impact of the secretome of MSCs from different tissue sources on neuronal differentiation and axonal growth. Biochimie 155: 83-91.

19. Francisco J Vizoso, Noemi Eiro, Luis Costa, Paloma Esparza, Mariana Landin, et al. (2019) Mesenchymal stem cells in homeostasis and systemic diseases: Hypothesis, evidences, and therapeutic opportunities. Int J Mole Sci 20: 3738.

20. Sylwia Dabrowska, Anna Andrzejewska, Barbara Lukomska, Miroslaw Janowski (2019) Neuroinflammation as a target for treatment of stroke using mesenchymal stem cells and extracellular vesicles. J Neuroinflammation 16: 1-17.

21. Andreia G Pinho, Jorge R Cibrão, Nuno A Silva, Susana Monteiro, António J Salgado (2020) Cell secretome: Basic insights and therapeutic opportunities for CNS disorders. Pharmaceuticals 12: 31 . 